\title{
Metastatic colorectal cancer: therapeutic options for treating refractory disease
}

\author{
A. Parmar MD, ${ }^{*}$ K.K.W. Chan MD MSc PhD, ${ }^{* \dagger}$ and Y.J. Ko MD MMSc SM*
}

\begin{abstract}
Therapeutic options for chemorefractory metastatic colorectal cancer (mcRc) have significantly expanded since 2009. The oral targeted therapies regorafenib and trifluridine/tipiracil have been established to be efficacious and safe in patients with mCRC who have progressed beyond 2 or more lines of chemotherapy. Evidence for the use of immunotherapy in a subgroup of this patient population is also encouraging, particularly in patients with mCRC that exhibits high microsatellite instability or deficient mismatch repair. Those significant advances have led to Health Canada approval of 3 novel therapeutic options for the treatment of patients with chemorefractory mcrc. However, the limited clinical efficacy of those treatments underscores the need for ongoing development of systemic therapy options for this unique cohort of patients. Here, we review the current and emerging treatment landscape for chemorefractory mCRC.
\end{abstract}

Key Words Colorectal cancer, metastatic, targeted therapy, immunotherapy, treatment-refractory disease

Curr Oncol. 2019 November;26(S1):S24-S32 www.current-oncology.com

\section{INTRODUCTION}

Colorectal cancer (CRC) is the 2 nd most common cancer in the Canadian population, with more than 25,000 patients diagnosed in $2017^{1}$. Although most diagnoses are made at an early stage, up to $50 \%$ of patients will develop metastatic disease $^{2,3}$. Unfortunately, despite advances in both chemotherapy and targeted therapy, survival in metastatic CRC (mCRC) remains poor, with the 5-year survival rate being $20 \%$ or less ${ }^{1}$. For select patients with liver metastases who are eligible for surgical resection, survival improves, with 5 -year survival rates reaching up to $50 \%{ }^{4-6}$. However, after surgical resection, most of those patients will ultimately develop recurrent disease, for which many will require further treatment with systemic therapy ${ }^{4,5,7}$.

Since the start of the 2000s, primary systemic therapy for unresectable mcRc has consisted of fluorouracil-based chemotherapy in combination with oxaliplatin or irinotecan, which has an associated overall survival (os) of up to 24 months $^{8,9}$. The addition of biologic therapy targeting either vascular endothelial growth factor (VEGF) or the epidermal growth factor receptor (EGFR - in RAS wild-type disease) to combination chemotherapy has further improved patient outcomes, with median os improvements reaching upwards of 30 months $^{10-12}$. However, despite those notable improvements in first- and second-line systemic therapies, many patients will develop progressive disease on those standard chemotherapy regimens, establishing the need for systemic therapy options in the chemorefractory setting (Figure 1).

\section{REVIEW}

\section{Targeted Therapy in Chemorefractory mCRC}

\section{Regorafenib}

Regorafenib is an oral tyrosine kinase inhibitor with targets in pathways important in angiogenesis (VEGF receptors $1-3$, Tie2), oncogenesis (C-KIT, RAF, BRAF), and the tumour microenvironment (platelet-derived growth factor receptor, fibroblast growth factor receptor ${ }^{13}$. Use of regorafenib in chemorefractory mCRC gained approval from the U.S. Food and Drug Administration (FDA) in 2012 and from Health Canada in 2017, after positive results were reported from the CORRECT trial ${ }^{14}$. That international phase III randomized controlled trial (RCT) evaluated the use of regorafenib in patients who had evidence of disease progression on all previously available systemic therapies, including fluorouracil, irinotecan, oxaliplatin, bevacizumab, and anti-EGFR therapy (for patients with 


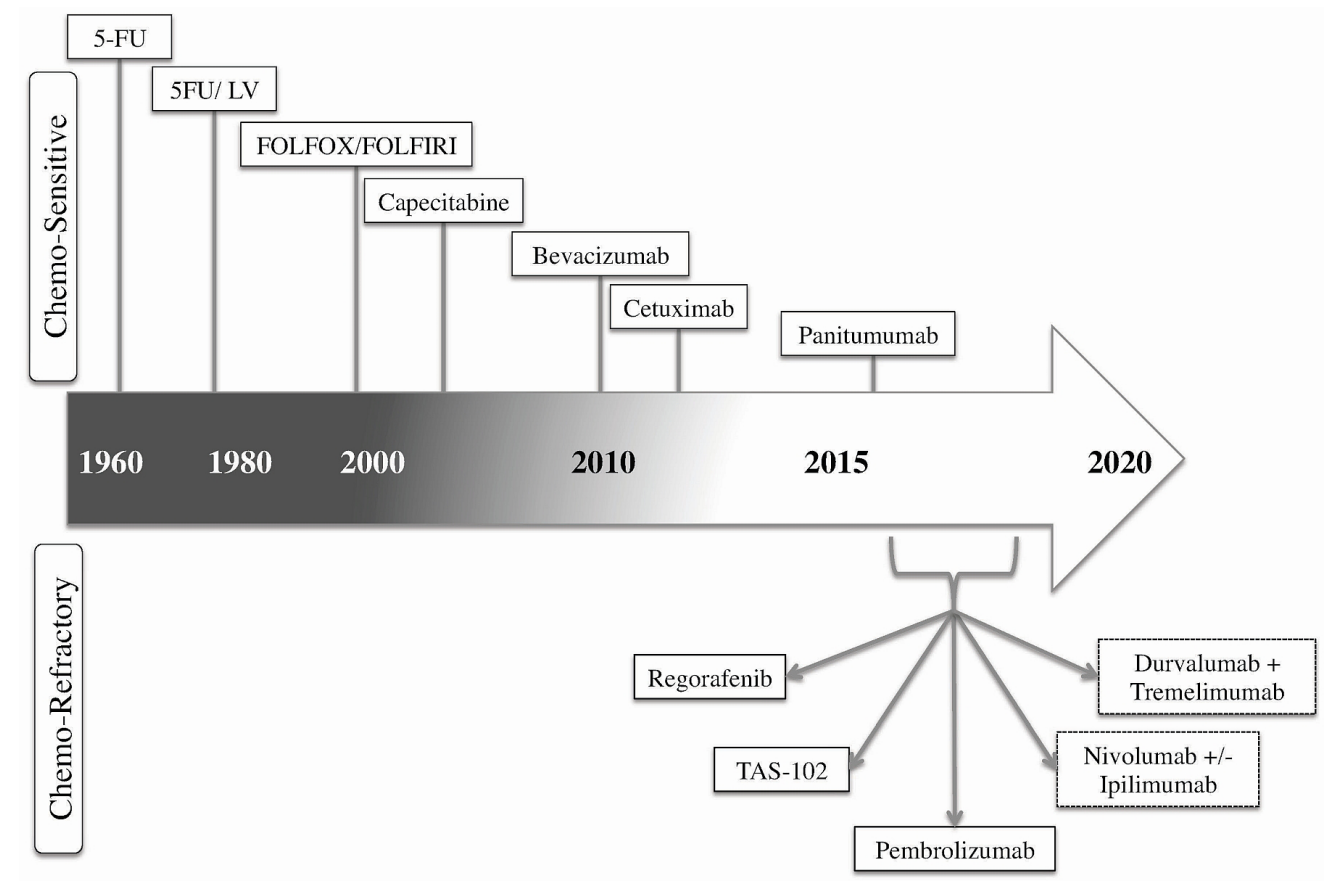

FIGURE 1 Evolution of the systemic therapy landscape for metastatic colorectal cancer, both chemosensitive and chemorefractory. In the chemorefractory setting, significant progress has been made in the form of both targeted agents and immunotherapy. A clinical need for treatment options in that setting has led to timely Health Canada approval for many of the novel agents (depicted in boxes with solid outlines). Early evidence for novel immunotherapy combinations (depicted in boxes with dotted outlines) offers the promise of further improvements in outcomes for this cohort of patients; however, more data are required before adoption can become widespread. 5-FU = 5-fluorouracil; LV = leucovorin; FOLFOX = leucovorin-fluorouracil-oxaliplatin; FOLFIRI = leucovorin-fluorouracil-irinotecan; TAS-102 = trifluridine/tipiracil.

$K R A S$ wild-type disease $)^{14}$. Patients were randomized in a 2:1 design to receive either oral regorafenib (160 $\mathrm{mg}$ for 21 days of a 28-day schedule) or placebo, both in conjunction with best supportive care. Treatment with regorafenib led to a significant improvement in progression-free survival [PFS: 1.9 months vs. 1.7 months; hazard ratio (HR): 0.49; $p<0.0001]$ and os (6.4 months vs. 5.0 months; HR: $0.77 ; p=$ 0.0052). Regorafenib did not demonstrate a significant benefit in the objective response rate (ORR), with no complete responses and only 5 partial responses (1\%). However, the significant benefit of regorafenib treatment compared with placebo was demonstrated in the achievement of disease control (defined as the combined rate of partial responses and stable disease): $41 \%$ compared with 15\%, $p<0.0001^{14}$ (Table I).

The CONCUR trial set out to evaluate the efficacy of regorafenib treatment in a primarily Asian population, recognizing that only $14 \%$ of patients in the CORRECT trial were of Asian heritage ${ }^{15}$. Although similar in design to CORRECT, CONCUR had no mandatory requirement for patients to have received prior treatment with anti-vEGF or (in $R A S$ wild-type disease) anti-EGFR therapy; as a result, $41 \%$ and $38 \%$ of randomized patients had not received those agents before enrolment. As in CORRECT, treatment with regorafenib in this Asian population led to improvements in PFS (3.2 months vs. 1.7 months; HR: $0.31 ; p<0.0001$ ) and os (8.8 months vs. 6.3 months; HR: $0.55 ; p=0.00016$; Table I $)^{15}$.

Although the positive results of CORRECT and CONCUR established the efficacy of regorafenib in a chemorefractory population, the high rate of adverse events (AEs) requires consideration before universal use of this agent can be recommended. The most common AEs associated with regorafenib treatment include hand-foot syndrome, hypertension, fatigue, and hepatotoxicity ${ }^{14,15}$. Treatment-related AEs of any grade were noted in more than $90 \%$ of patients treated with regorafenib in both trials, with grades 3 and 4 AEs occurring in more than $50 \%$ of patients. Those AEs necessitated treatment modification in up to $60 \%$ of patients receiving regorafenib ${ }^{14,15}$. However, treatment discontinuation because of AEs was infrequent ${ }^{14,15}$. Given that most AEs occur within the first 2 cycles of treatment, there is interest in the pursuit of dose reductions early in the treatment course to reduce the toxicities associated with regorafenib ${ }^{18}$; however, high-level evidence to support that approach is currently lacking.

Post-marketing surveillance studies have confirmed the efficacy and safety data reported in the CORRECT and CONCUR trials. The safety profile reported in Consign, an international trial of regorafenib in non-Asian patients was consistent with those in the two RCTs, with treatmentrelated AEs occurring in $91 \%$ of patients, and treatment discontinuations occurring in $9 \%^{19}$. In a French population, the REBECCA trial reported treatment-related AEs in more than $80 \%$ of patients ${ }^{20}$. Efficacy data from REBECCA demonstrated a median os duration of 5.6 months with regorafenib treatment ${ }^{20}$. Although the os duration in REBECCA was shorter than that seen in the CORRECT and CONCUR trials, the evaluated population included a subset of patients 
TABLE I Key phase III efficacy data for targeted therapies in chemorefractory metastatic colorectal cancer

\begin{tabular}{|c|c|c|c|c|c|c|c|c|}
\hline \multirow[t]{3}{*}{ Variable } & \multicolumn{4}{|c|}{ Trials of regorafenib } & \multicolumn{4}{|c|}{ Trials of trifluridine/tipiracil } \\
\hline & \multicolumn{2}{|c|}{ CORRECT $^{14}$} & \multicolumn{2}{|c|}{ CONCUR $^{15}$} & \multicolumn{2}{|c|}{ RECOURSE $^{16}$} & \multicolumn{2}{|c|}{ TERRA $^{17}$} \\
\hline & Treatment & Placebo & Treatment & Placebo & Treatment & Placebo & Treatment & Placebo \\
\hline Patients $(n)$ & 505 & 255 & 136 & 68 & 534 & 266 & 271 & 135 \\
\hline ORR $[n(\%)]$ & $5(1)$ & $1(0.4)$ & $6(4)$ & $0(0)$ & $8(1.6)$ & $1(0.4)$ & $1(1.1)$ & $0(0)$ \\
\hline$p$ Value & \multicolumn{2}{|c|}{0.19} & \multicolumn{2}{|c|}{$0.05^{\mathrm{a}}$} & \multicolumn{2}{|c|}{0.29} & \multicolumn{2}{|c|}{0.55} \\
\hline \multicolumn{9}{|l|}{ PFS (months) } \\
\hline Median & 1.9 & 1.7 & 3.2 & 1.7 & 2.0 & 1.7 & 2.0 & 1.8 \\
\hline IQR & $1.6-3.9$ & $1.4-1.9$ & $2.0-3.7$ & $1.6-1.8$ & $1.9-2.1$ & $1.7-1.8$ & $1.9-2.8$ & $1.7-1.8$ \\
\hline $\mathrm{HR}$ & \multicolumn{2}{|c|}{0.49} & \multicolumn{2}{|c|}{0.31} & \multicolumn{2}{|c|}{0.48} & \multicolumn{2}{|c|}{0.43} \\
\hline $95 \% \mathrm{Cl}$ & \multicolumn{2}{|c|}{0.42 to 0.58} & \multicolumn{2}{|c|}{0.22 to 0.44} & \multicolumn{2}{|c|}{0.41 to 0.57} & \multicolumn{2}{|c|}{0.34 to 0.54} \\
\hline$p$ Value & \multicolumn{2}{|c|}{$<0.0001$} & \multicolumn{2}{|c|}{$<0.0001^{\mathrm{a}}$} & \multicolumn{2}{|c|}{$<0.001$} & \multicolumn{2}{|c|}{$<0.001$} \\
\hline \multicolumn{9}{|l|}{ OS (months) } \\
\hline Median & 6.4 & 5.0 & 8.8 & 6.3 & 7.1 & 5.3 & 7.8 & 7.1 \\
\hline IQR & $3.6-11.8$ & $2.8-10.4$ & $7.3-9.8$ & $4.8-7.6$ & $6.5-7.8$ & $4.6-6.0$ & $7.1-8.8$ & $5.9-8.2$ \\
\hline $\mathrm{HR}$ & \multicolumn{2}{|c|}{0.77} & \multicolumn{2}{|c|}{0.55} & \multicolumn{2}{|c|}{0.68} & \multicolumn{2}{|c|}{0.79} \\
\hline $95 \% \mathrm{Cl}$ & \multicolumn{2}{|c|}{0.64 to 0.94} & \multicolumn{2}{|c|}{0.40 to 0.77} & \multicolumn{2}{|c|}{0.58 to 0.81} & \multicolumn{2}{|c|}{0.62 to 0.99} \\
\hline$p$ Value & \multicolumn{2}{|c|}{0.0052} & \multicolumn{2}{|c|}{$0.0002^{\mathrm{a}}$} & \multicolumn{2}{|c|}{$<0.001$} & & \\
\hline
\end{tabular}

a One-sided.

ORR = objective response rate; $\mathrm{PFS}=$ progression-free survival; IQR $=25 \%-75 \%$ interquartile range; $\mathrm{HR}=$ hazard ratio; $\mathrm{Cl}=$ confidence interval; OS = overall survival.

with a poorer performance status. Thus, those real-world effectiveness data provide supportive evidence of benefit for the use of regorafenib in the chemorefractory setting.

\section{Trifluridine/Tipiracil}

Trifluridine/tipiracil is an oral combination therapy consisting of a thymidine nucleoside analogue (trifluridine) and a thymidine phosphorylase inhibitor (tipiracil hydrochloride $)^{21}$. Trifluridine, the active component in this combination, is incorporated into DNA, where it exerts an antitumour effect; tipiracil increases the level of trifluridine by preventing its breakdown ${ }^{21}$. Approvals by the U.S. FDA and by Health Canada for the use of this agent in chemorefractory mCRC were granted in 2017 and 2018 respectively, after publication of the positive results of the RECOURSE trial ${ }^{16}$.

The international, double-blind, placebo-controlled RECOURSE trial evaluated the use of trifluridine/tipiracil in patients who had experienced progression while taking fluorouracil, oxaliplatin, irinotecan, bevacizumab, and (in $K R A S$ wild-type disease) EGFR-targeted therapy ${ }^{16}$. Prior regorafenib use was noted in $17 \%$ of the trifluridine/tipiracil group and in $20 \%$ of the placebo group. The 800 participating patients were randomized 2:1 to receive trifluridine/ tipiracil $35 \mathrm{mg} / \mathrm{m}^{2}$ twice daily on a 28 -day schedule (5 days of treatment and 2 days off for each of the first 2 weeks, followed by a 2-week rest period). After a median follow-up of 11.8 months, the group receiving trifluridine/tipiracil experienced an improvement in both PFs (2.0 months vs.
1.7 months; HR: $0.48 ; p<0.001)$ and os (7.1 months vs. 5.3 months; HR: $0.68 ; p<0.001)^{16}$. With long-term follow-up, ongoing evidence of an os benefit has been observed (HR: 0.69; $p<0.0001)^{22}$. As with regorafenib treatment, trifluridine/ tipiracil treatment, compared with placebo, was associated with a low ORR ( $1.6 \%$ vs. $0.4 \%, p=0.29)$; however, disease control (complete response, plus partial response, plus stable disease) was significantly improved ( $44 \%$ vs. $16 \%$, $p<0.001$, Table I $)^{16}$.

The TERRA phase III RCT evaluated the safety and efficacy of trifluridine/tipiracil in an Asian population ${ }^{17}$. Similar in design to RECOURSE, TERRA enrolled patients at 30 sites in China, Thailand, and the Republic of Korea. Trial enrolment was limited to patients with mcRc who had an Eastern Cooperative Oncology Group performance status of $0-1$ and who had evidence of progressive disease while receiving 2 prior chemotherapy regimens (fluorouracil, irinotecan, and oxaliplatin). Unlike the Recourse trial, TERRA did not require patients to have demonstrated progression on prior anti-vEGF or anti-EGFR therapy. Treatment with trifluridine/tipiracil was associated with a significantly improved os duration (median: 7.8 months vs. 7.1 months with placebo; HR: $0.79 ; p=0.035)$. A significant benefit in PFS was also demonstrated (2.0 months vs. 1.8 months; HR: $0.43 ; p<0.001$; Table $)^{17}$.

Adverse events were common with trifluridine/ tipiracil, with grades 3 and 4 AEs occurring in $69 \%$ of patients in the RECOURSE trial ${ }^{16}$. Myelosuppression was the most frequent grade 3 or $4 \mathrm{AE}$ associated with trifluridine/tipiracil, 
including anemia ( $18 \%$ vs. $3 \%$ with placebo), neutropenia $(38 \% \text { vs. } 0 \% \text { ), and thrombocytopenia ( } 5 \% \text { vs. }<1 \%)^{16}$. Anygrade febrile neutropenia was observed in $4 \%$ of patients treated with trifluridine/tipiracil, with 1 treatmentrelated death occurring secondary to septic shock. The occurrences of grades 3 and 4 nausea ( $2 \%$ vs. $1 \%$ ), vomiting ( $2 \%$ vs. $<1 \%$ ), and diarrhea ( $3 \%$ vs. $<1 \%$ ) were uncommon with trifluridine/tipiracil ${ }^{16}$. Grades 3 and 4 fatigue $(4 \%$ vs. $6 \%$ ), loss of appetite ( $4 \%$ vs. $5 \%)$, abdominal pain $(2 \%$ vs. $4 \%$ ), and liver enzyme elevations (alanine aminotransferase: $2 \%$ vs. $4 \%$; aspartate aminotransaminase: $4 \%$ vs. $6 \%$; alkaline phosphatase: $8 \%$ vs. $11 \%$; bilirubin: $9 \%$ vs. $12 \%$ ) were more common in the placebo group. Treatment-related AEs led to dose modifications in $14 \%$ of patients and treatment withdrawals in $4 \%^{16}$.

In the TERRA trial, grades 3 and 4 treatment-related AES were observed in $46 \%$ of patients (compared with $10 \%$ for those receiving placebo), with resultant dose reductions in $8.5 \%$ of patients and treatment withdrawal in $10 \%{ }^{17}$. Deaths attributable to treatment-related AEs were not observed in the TERRA trial.

\section{Sequencing of Oral Therapies}

To date, no direct comparison of regorafenib and trifluridine/tipiracil has been made. However, indirect comparative evidence for efficacy and safety has been generated for the two agents.

Abrahao et al. ${ }^{23}$ conducted a systematic review and network meta-analysis of all available phase III RCTS evaluating either trifluridine/tipiracil or regorafenib. Their analysis included the CORRECT and CONCUR trials for regorafenib and the RECOURSE trial for trifluridine/ tipiracil. The results of the indirect comparison revealed no significant differences between the two oral agents with respect to PFS [HR: $0.85 ; 95 \%$ confidence interval (CI): 0.40 to $1.81 ; p=0.67$ ] and os (HR: $0.96 ; 95 \%$ CI: 0.57 to $1.66 ; p=0.91)^{23}$. With respect to safety, regorafenib was associated with more AEs of any grade (risk difference: 0.35 ; $95 \%$ Сı: 0.04 to $0.67 ; p=0.013$ ) and of grades $3-5$ (risk difference: $0.22 ; 95 \%$ cI: 0.13 to $0.31 ; p<0.001)^{23}$. However, compared with trifluridine/tipiracil, regorafenib was associated with fewer hematologic toxicities and more hand-foot symptoms ${ }^{23}$.

Moriwaki et al. ${ }^{24}$ performed a retrospective cohort study and propensity score analysis to compare trifluridine/tipiracil with regorafenib in a primarily Japanese population. No difference in os was observed in the overall analysis. In a subgroup analysis, an interaction of age with os favoured regorafenib in younger patients (that is, $<65$ years), and a preferential survival benefit with trifluridine/ tipiracil was observed in older patients $(\geq 65$ years and older) ${ }^{24}$. However, given the retrospective evidence and limited generalizability, those results require confirmation with international and prospective data.

Overall, high-level evidence to inform the therapeutic choice between trifluridine/tipiracil and regorafenib is lacking. In the RECOURSE RCT, subgroup analyses did not demonstrate any negative influence of prior regorafenib use on os, thus providing indirect evidence for the efficacy of trifluridine/tipiracil after treatment with regorafenib ${ }^{16}$. The same evidence for the reverse sequence is lacking, because no patients in the trials of regorafenib had received prior trifluridine/tipiracil. Further data to guide the sequencing of these oral agents is therefore warranted. Until those data are available, treatment selection is best guided by the unique toxicity profiles of the two agents and patientclinician preference.

\section{Immunotherapy in Chemorefractory mCRC}

Given demonstrated clinical improvements in difficultto-treat tumours and the potential for long-term durable responses, there is significant interest in the use of immunotherapy for chemorefractory mCRC $^{25}$. Furthermore, a biologic rationale for that approach exists. For instance, the association of high immune cell infiltration in CRC with favourable outcomes suggests a potential role for the host immune system to mitigate carcinogenesis ${ }^{26}$. As well, high tumour mutational burden (TMB) has been associated with a positive response to immunotherapy ${ }^{27}$. In CRC, the presence or absence of intact DNA repair mechanisms with the DNA mismatch repair genes has allowed for the identification of unique signatures ${ }^{25}$ :

Deficient mismatch repair ( microsatellite instability (MSI-H) CRC characterized by a high тмв

- Proficient (рммк), leading to low microsatellite instability (MSI-L) or microsatellite stable (MSS) CRC characterized by a low тмв

High тмв and pathophysiologic correlative data of high immune-cell infiltrate in dMMR (MSI-H) tumours therefore creates an opportunity for a positive response to immunotherapy in the relevant patients.

\section{Immune Checkpoint Blockade}

In clinical trial evaluation of immunotherapy with the immune checkpoint blockade agents pembrolizumab (PD-1 blockade), nivolumab (PD-1 blockade), and combination nivolumab and ipilimumab (CTLA4 blockade), phase II evidence has demonstrated encouraging responses ${ }^{28}$ (Table II). In KEYNOTE-164, the use of pembrolizumab was evaluated in patients with chemorefractory ( 1 or more prior lines of chemotherapy) dMMR mCRC, pMMR mCRC, and dMMR cancers of any site. In the cohort of patients with dMMR mCRC, the oRR was $40 \%$, but no responses were noted in the cohort of patients with pMMr mCRC ${ }^{28}$. Long-term follow-up data in the cohort with dMMR mCRC revealed an ongoing response, with an ORR of $32 \%$ and a median duration of response not reached ${ }^{32}$. Survival analysis in the latter cohort found a median PFs of 4.1 months (95\% cI: 2.1 months to not reached) and a 12-month os rate of $76 \%^{32}$. Those positive results of KEYNOTE-164 and related studies led to approvals by both the U.S. FDA (2017) and Health Canada (2019) for the use of pembrolizumab in the population with dMMR (MSI-H) chemorefractory mCRC ${ }^{28,32}$.

The ongoing international nonrandomized phase II CheckMate 142 trial is evaluating the use of nivolumab monotherapy or nivolumab combination treatment in patients with MSI-H or MSI-L chemorefractory mCRC. Currently, only data for the cohorts with MSI-H mCRC receiving nivolumab monotherapy and nivolumab-ipilimumab have 
TABLE II Key phase II efficacy data for immunotherapy in chemorefractory metastatic colorectal cancer

\begin{tabular}{|c|c|c|c|c|c|c|c|}
\hline \multirow[t]{2}{*}{ Agent } & \multirow[t]{2}{*}{ Reference } & \multirow{2}{*}{$\begin{array}{l}\text { Population } \\
\text { (n) }\end{array}$} & \multirow{2}{*}{$\begin{array}{l}\text { Prior treatment } \\
\text { (n) }\end{array}$} & \multicolumn{3}{|c|}{ ORR $[n(\%)]$} & \multirow[t]{2}{*}{ PFS } \\
\hline & & & & CR & PR & SD & \\
\hline \multicolumn{8}{|c|}{ Pembrolizumab } \\
\hline \multirow{3}{*}{\multicolumn{2}{|c|}{ Le et al., $2015^{28}$}} & $18 \mathrm{pMMR}$ & Not specified & 0 & 0 & $2(11)$ & 2.2 Months \\
\hline & & & & & & & ( $95 \%$ Cl: 1.4 months to 2.8 months) \\
\hline & & $10 \mathrm{dMMR}$ & & 0 & $4(40)$ & $5(50)$ & Not specified \\
\hline \multicolumn{8}{|c|}{ Nivolumab } \\
\hline & Overman et al., $2017^{29}$ & 74 dMMR (MSI-H) & 12 Regorafenib & $2(3)$ & $22(30)$ & $25(34)$ & $50 \%$ at 12 months \\
\hline \multicolumn{8}{|c|}{ Nivolumab-ipilimumab } \\
\hline \multirow{2}{*}{\multicolumn{2}{|c|}{ Overman et al., $2018^{30}$}} & 119 dMMR (MSI-H) & 11 Regorafenib & $4(3)$ & $61(51)$ & $37(31)$ & $71 \%$ at 12 months \\
\hline & & & 2 Trifluridine/tipiracil & & & & \\
\hline \multicolumn{8}{|c|}{ Durvalumab-tremelimumab } \\
\hline \multirow{2}{*}{\multicolumn{2}{|c|}{ Chen et al., $2019^{31}$}} & 179 Unselected & $\begin{array}{l}\text { Regorafenib, } \\
\text { not specified }\end{array}$ & 0 & $1(1)$ & $26(22)$ & - \\
\hline & & & 0 Trifluridine/tipiracil & & & & \\
\hline
\end{tabular}

a In the chemorefractory setting.

$\mathrm{ORR}=$ objective response rate; $\mathrm{CR}=$ complete response; $\mathrm{PR}=$ partial response; $\mathrm{SD}=$ stable disease; $\mathrm{PFS}=$ progression-free survival; $\mathrm{pMMR}=$ mismatch repair proficient; $\mathrm{dMMR}=$ mismatch repair deficient; $\mathrm{MSI}-\mathrm{H}=$ high microsatellite instability.

been published ${ }^{29,30}$. In the nivolumab monotherapy arm, patients with MSI-H mCRC were treated with nivolumab ( $3 \mathrm{mg} / \mathrm{kg}$ every 2 weeks) until evidence of progressive disease or toxicity. Treatment with nivolumab resulted in an ORR of $31 \%$ by investigator assessment. At the 12-month follow-up, the PFs rate was $50 \%$ and the os rate was $73 \%$, with the median duration of response, PFs, and os not being reached ${ }^{29}$.

Published results from the cohort treated with nivolumab-ipilimumab in the MSI-H population have also been positive ${ }^{30}$. The 119 patients in that cohort were treated with nivolumab $3 \mathrm{mg} / \mathrm{kg}$ in combination with ipilimumab $1 \mathrm{mg} / \mathrm{kg}$ every 3 weeks for 4 cycles, followed by nivolumab $3 \mathrm{mg} / \mathrm{kg}$ every 2 weeks until disease progression or toxicity. Per investigator assessment, the oRR was 55\%, with most patients attaining a partial response $(51 \%)^{30}$. As in the cohort of patients treated with nivolumab monotherapy, median duration of response, PFs, and os in the nivolumabipilimumab cohort was not reached at a median follow-up of 13 months ${ }^{29,30}$. The PFs and os rates at 12 months were $71 \%$ and $85 \%$ respectively (Table $\mathrm{II})^{30}$.

Early evidence for the benefit of combination immune checkpoint blockade in a population unselected for mismatch repair was recently demonstrated in a phase II RCT. The Canadian Cancer Trials Group co.26 trial randomized 179 unselected patients with chemorefractory mCRC 2:1 to combination treatment with durvalumab (1500 mg) and tremelimumab (75 mg) every 3 weeks for 4 cycles, followed by durvalumab monotherapy every 28 days or to best supportive care ${ }^{31}$. Median os was 6.6 months in the combination arm compared with 4.1 months in best supportive care arm (HR: 0.72; $90 \%$ CI: 0.54 to $0.97 ; p=0.07)^{31}$. Based on the pre-specified significance level of $p<0.10$, those results represented a significant improvement in os with the use of combination immunotherapy. No significant difference in PFS was noted. However, the disease control rate was significantly higher in the combination arm (odds ratio: 4.16; $p=0.006$; Table II ${ }^{31}$.

With respect to safety, AE rates associated with the use of immune checkpoint blockade are high. In KEYNOTE-164, pembrolizumab treatment was associated with AEs of any grade in $98 \%$ of patients and with grade 3 or 4 AEs in $41 \%^{28}$. Treatment-related AEs included rash or pruritus $(24 \%)$, endocrine disturbances (10\%), and asymptomatic pancreatitis $(15 \%)^{28}$. Similarly, treatment with nivolumab monotherapy in the CheckMate 142 trial was associated with AEs of any grade in $70 \%$ of patients and grade 3 or 4 AEs in $21 \% 29$. The most frequent treatment-related AEs included fatigue $(22 \%)$, diarrhea $(20 \%)$, pruritus $(14 \%)$, and rash $(11 \%)$. Treatment discontinuation because of an AE was required in $7 \%$ of patients ${ }^{29}$. In both trials, no treatment-related deaths were noted. Combination nivolumab-ipilimumab led to grade 1 or 2 treatment-related AEs in $41 \%$ of patients, with grade 3 or 4 AEs occurring in $32 \%^{30}$. Treatment-related AEs necessitating discontinuation occurred in $13 \%$ of patients treated with the combination strategy ${ }^{30}$. Overall, the toxicity profile of immune checkpoint blockade either as monotherapy or as combination treatment in mCRC is similar to that seen with the use of those agents in other cancer disease sites ${ }^{33-36}$.

The foregoing results constitute promising evidence for the use of immunotherapy in dMMR (MSI-H) mCRC and of combination immunotherapy in an unselected population (Table II). However, definitive evidence in a phase III RCT evaluation of immunotherapy agents is warranted before 
those agents can be routinely recommended in chemorefractory mCRC, especially in the non-MSI-H population.

\section{Novel Immunotherapy Combination Strategies}

Combination strategies offer the promise of improved response with immunotherapy treatment. For instance, preclinical evidence has demonstrated a positive response with the combination of MEK inhibition and immune checkpoint blockade ${ }^{37}$. A phase I study investigating that approach with combination cobimetinib-atezolizumab reported an oRR of $8 \%$ in a predominantly mss population, leading to the development of a large phase III RCT to provide definitive evidence ${ }^{38}$.

The phase III IMblaze370 RCT randomized patients with mCRC and evidence of disease progression on 2 or more prior lines of chemotherapy to atezolizumab $(1200 \mathrm{mg}$ every 3 weeks) with or without cobimetinib (60 $\mathrm{mg}$ for 21 of every 28 days) or regorafenib ( $160 \mathrm{mg}$ for 21 of every 28 days $)^{39}$. Although all patients were eligible regardless of MsI status, the trial population $(n=363)$ had primarily MSI-L or Mss disease (90\%), given that enrolment of patients with MSI-H disease was capped at $5 \%$. At a median follow-up of 7 months, atezolizumab with or without cobimetinib was not found to be associated with improved os (8.87 months for atezolizumab-cobimetinib vs. 7.10 months for atezolizumab vs. 8.51 months for regorafenib) ${ }^{39}$. The stratified HR for atezolizumab-cobimetinib compared with regorafenib was 1.00 ( $p=0.99)$. No difference in ORR or PFs was demonstrated with the combination strategy ${ }^{39}$.

Combination strategies using DNA hypomethylating agents in conjunction with immune checkpoint blockade are speculated to improve immunogenicity by inducing gene expression of cancer-specific antigens ${ }^{40}$. However, despite the biologic rationale, the combination of the hypomethylating agent azacytidine with pembrolizumab was associated with a low oRR $(3 \%)$ in patients with chemorefractory Mss mcRC in a phase II trial ${ }^{40}$.

Despite those results, evaluation of combination strategies to sensitize and improve response to immunotherapy in both dMMr (MSI-H) and pMmR (MSI-L) disease is ongoing, with 16 phase II/III trials currently underway (Table III).

\section{Precision Medicine in Chemorefractory mCRC}

A better understanding of the molecular biology of CRC might allow for the application of rationally targeted therapies. Toward that end, the recognition of HER2 (human epidermal growth factor receptor 2) amplification in 3\%-4\% of CRC has led to interest in the evaluation of HER2-directed therapies in the metastatic setting ${ }^{41,42}$.

The proof-of-concept phase II HERACLEs trial evaluated trastuzumab in combination with lapatinib in chemorefractory KRAS wild-type mCRC ${ }^{43}$. The evaluated cohort was heavily pretreated, with $74 \%$ of patients having received 4 or more prior lines of therapy, including chemotherapy, anti-vEGF therapy, and anti-EGFR therapy. Of the 27 patients enrolled, 8 (30\%) achieved an objective response. Median PFS was 21 weeks, and the os rate at 1 year was $45 \%{ }^{43}$.

With such promising results in a heavily pretreated population, interest in the evaluation of HER2-directed therapy for mCRC is ongoing, with several phase II trials evaluating the approach, including the swog 1613 randomized phase II trial of combination pertuzumab-trastuzumab compared with combination cetuximab-irinotecan for unresectable mCRC that has progressed on prior chemotherapy (NCT03365882, NCT03457896, NCT03418558, and NCT03043313 at https://ClinicalTrials.gov/).

Molecular characterization of mCRC has also led to the identification of NTRK gene rearrangements in up to $2 \%$ of patients ${ }^{44,45}$. Recognition of the role that NTRK gene fusions take in the constitutive activation of oncogenic pathways has led to increasing interest in targeting those fusions in mCRC $^{45}$. Larotrectinib, an oral inhibitor of tropomyosin receptor kinases A-C, has demonstrated promising early-phase results in treatment-refractory gastrointestinal cancers, being associated with an ORR of $67 \%$ and an acceptable safety profile ${ }^{46}$. The early success of the drug has led to both U.S. FDA approval (2018) and conditional approval from Health Canada (2019). However, before widespread adoption of this agent for treatment-refractory mcRc, further efficacy data have to be established. Nevertheless, results so far highlight the significant potential that molecular characterization and rational targeting could hold for the treatment of patients with refractory mCRC.

\section{SUMMARY}

Significant progress in the management of mcrc has been made since the start of the 2000s, but effective therapeutic options for patients who ultimately progress on firstand second-line therapies are still lacking. Fortunately, the therapeutic landscape in chemorefractory mcRc has undergone significant modification since 2009, resulting in the timely approval of 3 systemic therapy options for this heavily pretreated patient population. However, the notably frequent toxicities associated with oral targeted therapies and the lack of a universal response to immunotherapy necessitates the further development of therapies that provide tolerable treatment options for the larger cohort of patients with mCRC. Novel combination strategies to help improve and sustain the response to immunotherapy have demonstrated early signs of efficacy for MSI-H and MSI-L or MSS mCRC. Furthermore, a better understanding of the molecular biology of mCRC creates opportunities to apply precision approaches involving rational directed therapies. Given those significant advances in therapeutic approaches, the world of gastrointestinal oncology looks forward to the ongoing development of systemic therapies that will be effective in the treatment-refractory setting.

\section{CONFLICT OF INTEREST DISCLOSURES}

We have read and understood Current Oncology's policy on disclosing conflicts of interest, and we declare the following interests: AP has received honoraria from Oncology Education. YJK has been a member of advisory boards for Taiho, Merck, and Bayer Pharmaceuticals and has also received research funding from Taiho. KKWC has no conflicts of interest to disclose.

\section{AUTHOR AFFILIATIONS}

*Odette Cancer Centre, Sunnybrook Health Sciences Centre, and ${ }^{\dagger}$ Canadian Centre for Applied Research in Cancer Control, Toronto, ON. 
CHEMOREFRACTORY METASTATIC COLORECTAL CANCER, Parmar et al.

TABLE III Ongoing phase II/III clinical trials in North America evaluating novel therapeutic strategies for chemorefractory metastatic colorectal cancer

\begin{tabular}{|c|c|c|c|c|}
\hline Agent & ClinicalTrials.gov ID & Phase & Population & Investigational strategy \\
\hline \multicolumn{5}{|c|}{ Targeted therapy combinations } \\
\hline & 02928224 & III & BRAF V600E mutated & Encorafenib-cetuximab with or without binimetinib \\
\hline & 02393755 & II & Unselected & Capecitabine-nintedanib \\
\hline & 03446157 & II & $\begin{array}{c}K R A S, \text { NRAS, } \\
B R A F \text { wild-type }\end{array}$ & Cetuximab-palbociclib \\
\hline
\end{tabular}

Immunotherapy with targeted therapy

\begin{tabular}{cccc} 
NCT03377361 & II & Unselected & $\begin{array}{c}\text { After prior doublet chemotherapy: nivolumab-trametinib } \\
\text { After prior triplet chemotherapy: nivolumab-ipilimumab-trametinib }\end{array}$ \\
\hline NCT03475004 & II & Unselected & Pembrolizumab-bevacizumab-binimetinib \\
\hline NCT03403634 & II & Unselected & Interferon-alfa-rintatolimod-celecoxib \\
NCT02860546 & II & Microsatellite-stable & Nivolumab-trifluridine/tipiracil \\
NCT03800602 & II & Microsatellite-stable & Nivolumab-metformin
\end{tabular}

Immunotherapy combinations

$\begin{array}{cccc}\text { NCT03473925 } & \text { II } & \text { Microsatellite-stable } & \text { Pembrolizumab-navarixin } \\ \text { NCT02981524 } & \text { II } & \text { pMMR } & \text { Pembrolizumab-GVAX }\end{array}$

Immunotherapy with chemotherapy

$\begin{array}{cccc}\text { NCT03832621 } & \text { II } & \begin{array}{c}\text { Microsatellite-stable } \\ \text { and MGMT silenced }\end{array} & \text { Nivolumab-ipilimumab-temozolomide } \\ \text { NCT02873195 } & \text { II } & \text { Unselected } & \text { Atezolizumab with or without capecitabine-bevacizumab }\end{array}$

Immunotherapy with radiation therapy

\begin{tabular}{cccc} 
NCT02437071 & II & Unselected & Pembrolizumab with RT or RFA \\
NCT03122509 & II & Unselected & Durvalumab-tremelimumab with RT or RFA \\
NCT03007407 & II & Microsatellite-stable & Durvalumab-tremelimumab with RT \\
NCT02888743 & II & Microsatellite-stable & Durvalumab-tremelimumab with RT \\
\hline
\end{tabular}

a Colon cancer vaccine (Aduro Biotech, Berkeley, CA, U.S.A.).

$\mathrm{pMMR}=$ mismatch repair proficient; MGMT $=\mathrm{O}^{6}$-methylguanine-DNA methyltransferase; $\mathrm{RT}=$ radiation therapy; RFA = radiofrequency ablation.

\section{REFERENCES}

1. Canadian Cancer Statistics Advisory Committee. Canadian Cancer Statistics 2018. Toronto, ON: Canadian Cancer Society; 2018.

2. Qiu M, Hu J, Yang D, Cosgrove DP, Xu R. Pattern of distant metastases in colorectal cancer: a SEER based study. Oncotarget 2015;6:38658-66.

3. Hugen N, van de Velde CJ, de Wilt JH, Nagtegaal ID. Metastatic pattern in colorectal cancer is strongly influenced by histological subtype. Ann Oncol 2014;25:651-7.

4. Abbas S, Lam V, Hollands M. Ten-year survival after liver resection for colorectal metastases: systematic review and meta-analysis. ISRN Oncol 2011;2011:763245.

5. Choti MA, Sitzmann JV, Tiburi MF, et al. Trends in long-term survival following liver resection for hepatic colorectal metastases. Ann Surg 2002;235:759-66.

6. Nordlinger B, Sorbye H, Glimelius B, et al. on behalf of the European Organisation for Research and Treatment of Cancer (воRTC) Gastro-Intestinal Tract Cancer Group, Cancer Research UK, the Arbeitsgruppe Lebermetastasen und-tumoren in der Chirurgischen Arbeitsgemeinschaft
Onkologie, the Australasian Gastro-Intestinal Trials Group, and the Fédération Francophone de Cancérologie Digestive. Perioperative Folfox 4 chemotherapy and surgery versus surgery alone for resectable liver metastases from colorectal cancer (EORTC 40983): long-term results of a randomised, controlled, phase 3 trial. Lancet Oncol 2013;14:1208-15.

7. Abdalla EK, Vauthey JN, Ellis LM, et al. Recurrence and outcomes following hepatic resection, radiofrequency ablation, and combined resection/ablation for colorectal liver metastases. Ann Surg 2004;239:818-25.

8. Goldberg RM, Sargent DJ, Morton RF, et al. A randomized controlled trial of fluorouracil plus leucovorin, irinotecan, and oxaliplatin combinations in patients with previously untreated metastatic colorectal cancer. J Clin Oncol 2004;22:23-30.

9. Tournigand C, Andre T, Achille E, et al. Folfiri followed by FOLFOX6 or the reverse sequence in advanced colorectal cancer: a randomized GERCOR study. JClin Oncol 2004;22:229-37.

10. Douillard JY, Siena S, Cassidy J, et al. Randomized, phase III trial of panitumumab with infusional fluorouracil, leucovorin, and oxaliplatin (FOLFOX4) versus FOLFOX4 alone as first-line treatment in patients with previously untreated 
metastatic colorectal cancer: the PRIME study. J Clin Oncol 2010;28:4697-705.

11. Hurwitz H, Fehrenbacher L, Novotny W, et al. Bevacizumab plus irinotecan, fluorouracil, and leucovorin for metastatic colorectal cancer. N Engl J Med 2004;350:2335-42.

12. Van Cutsem E, Kohne CH, Hitre E, et al. Cetuximab and chemotherapy as initial treatment for metastatic colorectal cancer. N Engl J Med 2009;360:1408-17.

13. Goel G. Evolution of regorafenib from bench to bedside in colorectal cancer: is it an attractive option or merely a "me too" drug? Cancer Manag Res 2018;10:425-37.

14. Grothey A, Van Cutsem E, Sobrero A, et al. on behalf of the CORRECT Study Group. Regorafenib monotherapy for previously treated metastatic colorectal cancer (CORRECT): an international, multicentre, randomised, placebo-controlled, phase 3 trial. Lancet 2013;381:303-12.

15. Li J, Qin S, Xu R, et al. on behalf of the concur investigators. Regorafenib plus best supportive care versus placebo plus best supportive care in Asian patients with previously treated metastatic colorectal cancer (CONCUR): a randomised, doubleblind, placebo-controlled, phase 3 trial. Lancet Oncol 2015;16:619-29.

16. Mayer RJ, Van Cutsem E, Falcone A, et al. on behalf of the Recourse Study Group. Randomized trial of TAS-102 for refractory metastatic colorectal cancer. $N$ Engl J Med 2015;372:1909-19.

17. Xu J, Kim TW, Shen L, et al. Results of a randomized, doubleblind, placebo-controlled, phase III trial of trifluridine/tipiracil (TAS-102) monotherapy in Asian patients with previously treated metastatic colorectal cancer: the TERRA study. J Clin Oncol 2018;36:350-8.

18. Bekaii-Saab TS, Ou FS, Anderson DM, et al. Regorafenib dose optimization study (ReDos): randomized phase iI trial to evaluate dosing strategies for regorafenib in refractory metastatic colorectal cancer (mCrC): an ACCRU Network study [abstract 611]. J Clin Oncol 2018;36:. [Available online at: https://ascopubs.org/doi/abs/10.1200/JCO.2018.36.4_suppl .611; cited 29 August 2019]

19. Van Cutsem E, Martinelli E, Cascinu S, et al. Regorafenib for patients with metastatic colorectal cancer who progressed after standard therapy: results of the large, single-arm, open-label phase IIIB Consign study. Oncologist 2019; 24:185-92.

20. Adenis A, de la Fouchardiere C, Paule B, et al. Survival, safety, and prognostic factors for outcome with regorafenib in patients with metastatic colorectal cancer refractory to standard therapies: results from a multicenter study (REBECCA) nested within a compassionate use program. BMC Cancer 2016;16:412. [Erratum in: BMC Cancer 2016;16:518]

21. Lenz HJ, Stintzing S, Loupakis F. TAS-102, a novel antitumor agent: a review of the mechanism of action. Cancer Treat Rev 2015;41:777-83.

22. Van Cutsem E, Mayer RJ, Laurent S, et al. on behalf of the RECOURSE Study Group. The subgroups of the phase III RECOURSE trial of trifluridine/tipiracil (TAS-102) versus placebo with best supportive care in patients with metastatic colorectal cancer. Eur J Cancer 2018;90:63-72.

23. Abrahao ABK, Ko YJ, Berry S, Chan KKW. A comparison of regorafenib and TAS-102 for metastatic colorectal cancer: a systematic review and network meta-analysis. Clin Colorectal Cancer 2018;17:113-20.

24. Moriwaki T, Fukuoka S, Taniguchi H, et al. Propensity score analysis of regorafenib versus trifluridine/tipiracil in patients with metastatic colorectal cancer refractory to standard chemotherapy (REGOTAS): a Japanese Society for Cancer of the Colon and Rectum Multicenter Observational Study. Oncologist 2018;23:7-15.
25. Ganesh K, Stadler ZK, Cercek A, et al. Immunotherapy in colorectal cancer: rationale, challenges and potential. Nat Rev Gastroenterol Hepatol 2019;16:361-75.

26. Galon J, Costes A, Sanchez-Cabo F, et al. Type, density, and location of immune cells within human colorectal tumors predict clinical outcome. Science 2006;313:1960-4.

27. Chan TA, Yarchoan M, Jaffee E, et al. Development of tumor mutation burden as an immunotherapy biomarker: utility for the oncology clinic. Ann Oncol 2019;30:44-56.

28. Le DT, Uram JN, Wang H, et al. PD-1 blockade in tumors with mismatch-repair deficiency. NEnglJ Med 2015;372:2509-20.

29. Overman MJ, McDermott R, Leach JL, et al. Nivolumab in patients with metastatic DNA mismatch repair-deficient or microsatellite instability-high colorectal cancer (CheckMate 142): an open-label, multicentre, phase 2 study. Lancet Oncol 2017;18:1182-91.

30. Overman MJ, Lonardi S, Wong KYM, et al. Durable clinical benefit with nivolumab plus ipilimumab in DNA mismatch repair-deficient/microsatellite instability-high metastatic colorectal cancer. J Clin Oncol 2018;36:773-9.

31. Chen EX, Jonker DJ, Kennecke HJ, etal. cCTG co.26 trial: a phase II randomized study of durvalumab (D) plus tremelimumab (T) and best supportive care (BSC) versus BsC alone in patients (pts) with advanced refractory colorectal carcinoma (rCRC) [abstract 481].JClin Oncol2019;37:. [Available online at: https://ascopubs. org/doi/abs/10.1200/JCO.2019.37.4_suppl.481;cited29August2019]

32. Le DT, Kavain P, Kim TW, et al. KeYNOTE-164: pembrolizumab for patients with advanced microsatellite instability high (MSI-H) colorectal cancer [abstract 3514]. J Clin Oncol 2018;36: [Available online at: https://ascopubs.org/doi/ abs/10.1200/JCO.2018.36.15_suppl.3514; cited 29 August 2019]

33. Garon EB, Rizvi NA, Hui R, et al. on behalf of the KEYNOTE-001 investigators. Pembrolizumab for the treatment of nonsmall-cell lung cancer. N Engl J Med 2015;372:2018-28.

34. Motzer RJ, Tannir NM, McDermott DF, et al. on behalf of the CheckMate 214 investigators. Nivolumab plus ipilimumab versus sunitinib in advanced renal-cell carcinoma. $N$ Engl $\mathrm{J}$ Med 2018;378:1277-90.

35. Robert C, Schachter J, Long GV, et al. on behalf of the KEYNOTE-006 investigators. Pembrolizumab versus ipilimumab in advanced melanoma. $N$ Engl J Med 2015;372:2521-32.

36. WolchokJD, Rollin L, Larkin J. Nivolumab and ipilimumab in advanced melanoma. NEnglJMed 2017;377:2503-4. [Erratum in: N Engl J Med 2018;379:2185]

37. Ebert PJR, Cheung J, Yang Y, et al. MAP kinase inhibition promotes $T$ cell and antitumour activity in combination with PD-L1 checkpoint blockade. Immunity 2016;44:609-21.

38. Bendell JC, Bang YJ, Chee CE, et al. A phase Iв study of safety and clinical activity of atezolizumab and cobimetinib in patients with metastatic colorectal cancer [abstract 560]. J Clin Oncol 2018;36:. [Available online at: https://ascopubs. org/doi/10.1200/JCO.2018.36.4_suppl.560; cited 29 August 2019]

39. Eng C, Kim TW, Bendell J, et al. On behalf of the IMblaze370 investigators. Atezolizumab with or without cobimetinib versus regorafenib in previously treated metastatic colorectal cancer (IMblaze370): a multicentre, open-label, phase 3, randomised, controlled trial. Lancet Oncol 2019;20:849-61.

40. Lee JJ, Sun W, Bahary N, etal. Phase 2 study of pembrolizumab in combination with azacitadine in subjects with metastatic colorectal cancer [abstract 3054]. J Clin Oncol 2017;35: [Available online at: https://ascopubs.org/doi/10.1200/ JCO.2017.35.15_suppl.3054; cited 29 August 2019]

41. Ross JS, Fakih M, Ali SM, et al. Targeting HER2 in colorectal cancer: the landscape of amplification and short variant mutations in ERBB2 and ERBB3. Cancer 2018;124:1358-73.

42. Richman SD, Southward K, Chambers P, et al. HER2 overexpression and amplification as a potential therapeutic target in 
colorectal cancer: analysis of 3256 patients enrolled in the QUASAR, FOCUS and PICCOLO colorectal cancer trials. J Pathol 2016;238:562-70.

43. Sartore-Bianchi A, Trusolino L, Martino C, et al. Dual-targeted therapy with trastuzumab and lapatinib in treatmentrefractory, KRAS codon 12/13 wild-type, HER2-positive metastatic colorectal cancer (HERACLES): a proof-of-concept, multicentre, open-label, phase 2 trial. Lancet Oncol 2016; $17: 738-46$.
44. Cocco E, Scaltriti M, Drilon A. NTRK fusion-positive cancers and Trk inhibitor therapy. Nat Rev Clin Oncol 2018;15:731-47.

45. Ricciuti B, Genova C, Crino L, Libra M, Leonardi GC. Antitumour activity of larotrectinib in tumors harboring NTRK gene fusions: a short review on the current evidence. Onco Targets Ther 2019;12:3171-9.

46. Drilon A, Laetsch TW, Kummar S, et al. Efficacy of larotrectinib in TRK fusion-positive cancers in adults and children. N Engl J Med 2018;378:731-9. 